\title{
STW 5 is effective in dextran sulfate sodium-induced colitis in rats
}

\author{
Walaa Wadie • Heba Abdel-Aziz • Hala F. Zaki • \\ Olaf Kelber • Dieter Weiser • Mohamed T. Khayyal
}

Accepted: 13 April 2012 / Published online: 6 May 2012

(C) The Author(s) 2012. This article is published with open access at Springerlink.com

\begin{abstract}
Purpose An herbal preparation, STW 5, used clinically in functional dyspepsia and irritable bowel syndrome, has been shown to possess properties that may render it useful in inflammatory bowel disease (IBD). The present work was conducted to study its effectiveness in a rat model of IBD. Methods An experimental model reflecting ulcerative colitis in man was adopted, whereby colitis was induced in Wistar rats by feeding them $5 \%$ dextran sulfate sodium (DSS) in drinking water for one week. STW 5 and sulfasalazine (as a reference standard) were administered orally daily for 1 week before colitis induction and continued during DSS feeding. The animals were then sacrificed, and the severity of colitis was evaluated macroscopically and microscopically. Colon samples were homogenized for determination of reduced glutathione, tumor necrosis factor- $\alpha$, and cytokineinduced neutrophil chemoattractant-3 as well as myeloperoxidase, glutathione peroxidase, and superoxide dismutase. In addition, colon segments were suspended in an organ bath to test their reactivity towards carbachol, $\mathrm{KCl}$, and trypsin.
\end{abstract}

\footnotetext{
W. Wadie $\cdot$ H. F. Zaki $\cdot$ M. T. Khayyal $(\bowtie)$

Department of Pharmacology, Faculty of Pharmacy,

Cairo University,

Cairo, Egypt

e-mail: mtkhayyal@gmail.com

H. Abdel-Aziz

Department of Pharmacology,

Institute of Pharmaceutical Chemistry, University of Münster,

Münster, Germany

O. Kelber $\cdot$ D. Weiser

Scientific Department, Steigerwald Arzneimittelwerk GmbH,

Darmstadt, Germany
}

Results STW 5 and sulfasalazine were both effective in preventing the shortening of colon length and the increase in both colon mass index and total histology score as well as the changes in biochemical parameters measured except changes in dismutase activity. DSS-induced colitis led to marked depression in colonic responsiveness to the agents tested ex vivo, an effect which was normalized by both drugs.

Conclusions The findings point to a potential usefulness of STW 5 in the clinical setting of ulcerative colitis.

Keywords DSS colitis - Colonic responsiveness · STW 5 . Cytokines

\section{Introduction}

Inflammatory bowel disease (IBD) in man is usually manifested either as ulcerative colitis (UC) or as Crohn's disease. In both conditions, the inflammatory processes in the gut are associated with disturbances in gastrointestinal motility [1, 2]. One animal model of UC involves feeding dextran sulfate sodium (DSS) in drinking water to rodents [3, 4]. The model is widely used for screening purposes because of its ease of induction, reproducibility, well-characterized mucosal injury [3], and its responsiveness to effective agents such as sulfasalazine. The aims of management of IBD are the induction and maintenance of remission. However, no maintenance medication has been shown to be completely effective for preventing relapse. The search, therefore, continues in the hope of finding effective and relatively nontoxic agents that could be useful in prolonging remission intervals while minimizing adverse reactions.

STW 5 consists of a fixed dose combination of wellcharacterized and standardized nine herbal extracts, namely 
bitter candytuft (Iberis amara), melissa leaf (Melissa officinalis), chamomile flower (Matricaria recutita), caraway fruit (Carum carvi), peppermint leaf (Mentha piperita), Angelica root (Angelica archangelica), milk thistle (Silybum marianum), greater celandine herb (Chelidonium majus) and liquorice root (Glycyrrhiza glabra) that has proved to be effective against functional gastrointestinal disorders such as functional dyspepsia and irritable bowel syndrome [5-7]. Khayyal et al. [8, 9] reported on its anti-ulcerogenic and mucosal protective effects and related at least part of its usefulness to its anti-inflammatory properties, a fact that prompted us to further explore the potential benefits of the drug in the DSS model of IBD.

\section{Materials and methods}

\section{Drugs}

STW 5 was provided in the form of its commercial preparation (Iberogast ${ }^{\mathbb{B}}$ ) by Steigerwald Arzneimittelwerk GmbH, Darmstadt, Germany. The preparation is provided as a tincture containing $31 \%$ alcohol. Sulfasalazine was obtained from El-Kahira Pharmaceutical Company, Cairo, Egypt and was used as a suspension in $1 \%$ methylcellulose. The concentration of all drugs was adjusted so that the required dose per $200 \mathrm{~g}$ of rat was found in $1 \mathrm{~mL}$ of solution/ suspension.

\section{Animals}

Adult male Wistar rats, weighing 150-200 g each, were obtained from the Modern Veterinary Office for Laboratory Animals, Cairo, Egypt and were left to acclimatize for 1 week before subjecting them to experimentation. They were provided with a standard pellet diet and were given water ad libitum. The animals were kept at a temperature of $22 \pm 3^{\circ} \mathrm{C}$ and a 12 -h light/dark cycle as well as a constant relative humidity throughout the experimental period. The study was approved by the Ethical Committee for Animal Experimentation at the Faculty of Pharmacy, Cairo University, Cairo, Egypt.

Induction of colitis

Colitis was induced by adding $5 \%$ DSS (molecular weight of 37-40 kD; TdB Consultancy, Uppsala, Sweden) to the drinking water, ad libitum for 7 days $[3,4]$.

\section{Experimental design}

Two sets of experiments were carried out: one with a view to determine changes in histological and biochemical parameters and one to study motility changes ex vivo.
Study of effect of STW 5 on histological and biochemical parameters

Rats were randomly assigned to seven groups of ten animals each as follows: two control groups given $31 \%$ ethanol (vehicle of STW 5), four STW 5-treated groups (0.5, 1, 2, and $5 \mathrm{~mL} / \mathrm{kg})$, and one sulfasalazine-treated group $(300 \mathrm{mg} /$ $\mathrm{kg}$ ). The drugs were administered orally once per day for 1 week while keeping the animals on a normal pellet diet and water ad libitum. DSS (5\%) was then added to the drinking water of all groups except one of the control groups (considered as normal control, not receiving DSS). DSS treatment was continued for 1 week while keeping the rats under the same drug regimen during that time. Animals were weighed just before DSS treatment and just before autopsy to determine whether colitis had an effect on body weight.

Twenty-four hours after the last dose of treatment, the rats were sacrificed by cervical dislocation. The colon was excised and its length measured. The colon was opened longitudinally, rinsed in ice-cold normal saline, cleaned of fat and mesentery, blotted on filter paper, and weighed. The ratio of colon weight in milligrams to total body weight in grams was taken as the colon mass index and was used as a measure of the degree of colonic edema and severity of inflammation. The colon was then cut longitudinally into two parts. One specimen was fixed in $10 \%$ formalin and preserved for histological examination, and the other was homogenized in ice-cold normal saline to obtain a $10 \%$ homogenate for assessment of the chosen biochemical parameters.

Determination of biochemical parameters The colon homogenate was divided into four aliquots for determining the following parameters:

Reduced glutathione One aliquot was deproteinized with icecold $5 \%$ sulfosalicylic acid then centrifuged at 3,000 rpm for $20 \mathrm{~min}$. The supernatant was used for spectrophotometric estimation of glutathione (GSH) [10].

Myeloperoxidase activity An aliquot was mixed with an equal volume of $100 \mathrm{mmol} / \mathrm{L}$ phosphate buffer $\mathrm{pH} 6$ containing $1 \%$ hexadecyltrimethylammonium bromide. The mixture was freeze-thawed, sonicated for $10 \mathrm{~s}$ and centrifuged at $10,000 \mathrm{rpm}$ for $15 \mathrm{~min}$ at $4^{\circ} \mathrm{C}$. The supernatant was used for spectrophotometric estimation of myeloperoxidase (MPO) activity [11].

Tumor necrosis factor alpha and cytokine-induced neutrophil chemoattractant-3 An aliquot was centrifuged at 13 $000 \mathrm{rpm}$ for 30 minutes at $4^{\circ} \mathrm{C}$. The supernatant was used for assaying tumor necrosis factor (TNF)- $\alpha$ and cytokineinduced neutrophil chemoattractant (CINC)-3 using specific 
enzyme-linked immunosorbent assay kits (R\&D Systems GmbH, Wiesbaden, Germany).

Superoxide dismutase and glutathione peroxidase An aliquot was ultra-centrifuged at $105000 \mathrm{~g}$ for 15 minutes at $4^{\circ} \mathrm{C}$. The supernatant was used for the assay of superoxide dismutase (SOD) and glutathione peroxidase (GPx) using specific spectrophotometric kits (Biodiagnostic, Giza, Egypt).

Histopathological examination Transverse sections, 4-6 $\mu \mathrm{m}$ in size, were prepared from paraffin-embedded colon segments from each animal of all experimental groups except those treated with the two lower doses of STW 5. The sections were stained with hematoxylin and eosin (H\&E) and examined under a light microscope. They were graded individually by a pathologist blinded to the treatment regimen. Each section was assigned a damage score between 0 and 3 for each of five parameters, namely, necrosis of epithelium, inflammatory infiltrate in lamina propria, inflammatory infiltrate in submucosa, dilatation of crypts, and submucosal edema.

The scores for the five parameters measured for each rat were summed to obtain the "total histology score," being maximally 15 (three being the maximum for the five parameters examined). The data were then represented using a box plot.

\section{Colonic responsiveness ex vivo}

Rats were allocated to four groups of animals, each comprising six rats: two control groups, one group treated with sulfasalazine $(300 \mathrm{mg} / \mathrm{kg})$ and one group with STW 5 $(5 \mathrm{~mL} / \mathrm{kg})$. They were subjected to the same treatment procedure as above.

After sacrifice, segments of the colon approximately $20 \mathrm{~mm}$ in length were taken $3 \mathrm{~cm}$ from the anal orifice and suspended longitudinally under a $1-\mathrm{g}$ load in a $30-\mathrm{mL}$ water jacketed organ bath containing Krebs-Henseleit solution of the following composition (millimole per liter): $\mathrm{NaCl}, 119.0 ; \mathrm{KCl}, 4.7 ; \mathrm{CaCl}_{2}, 2.5 ; \mathrm{MgSO}_{4}, 1.2 ; \mathrm{KH}_{2} \mathrm{PO}_{4}$, 1.2; $\mathrm{NaHCO}_{3}, 25.0$; glucose, 5.5. The bath was maintained at $37^{\circ} \mathrm{C}$ and aerated with carbogen.

The preparation was allowed to equilibrate for $1 \mathrm{~h}$. Isotonic contractions were recorded for carbachol $(0.03-1 \mu \mathrm{mol} / \mathrm{L})$ and $\mathrm{KCl}(9.09-72.7 \mathrm{mmol} / \mathrm{L})$. The relaxant effect of a protease-activated receptor (PAR)-2 agonist, trypsin (MP Biomedicals Inc., Solon, USA) was evaluated on a muscle strip pre-contracted with a submaximal dose of carbachol. Trypsin $(30 \mu \mathrm{mol} / \mathrm{L})$ was applied $5 \mathrm{~min}$ after the addition of carbachol and the extent of relaxation measured.

Statistical analysis

SPSS 17.0 was used for data management and data analysis. Mean and standard error described continuous variables

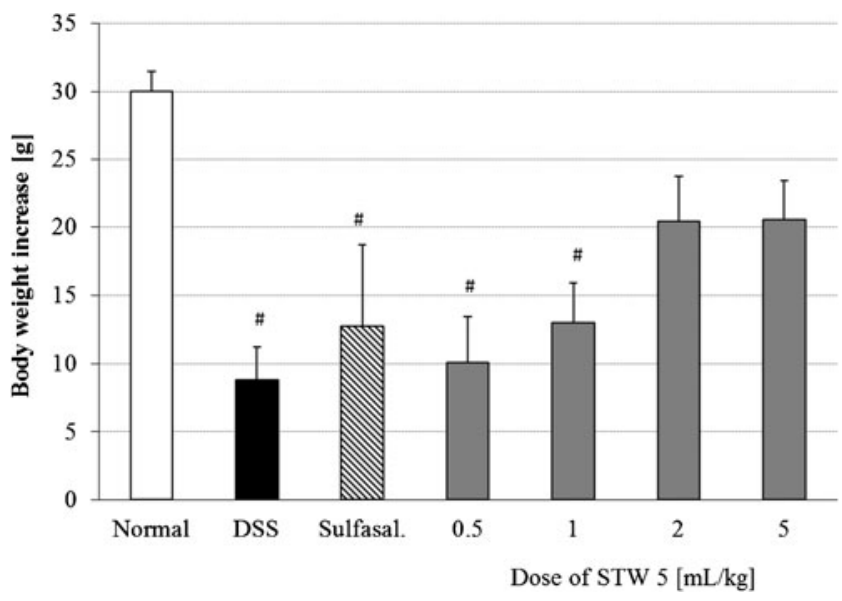

Fig 1 Effect of pretreatment with STW 5 on the increase in body weight of animals with DSS-induced colitis measured from the time of induction of colitis until sacrifice. Data are expressed as means \pm SEM of at least eight animals. ${ }^{\#} P \leq 0.05$ vs. normal control

with median and range for discrete data. Parametric and non-parametric ANOVA compared means and medians of different study groups. When significant, pair-wise comparisons were done using most restrictive tests: Scheffe test. $P$ value was considered significant at 0.05 or less.
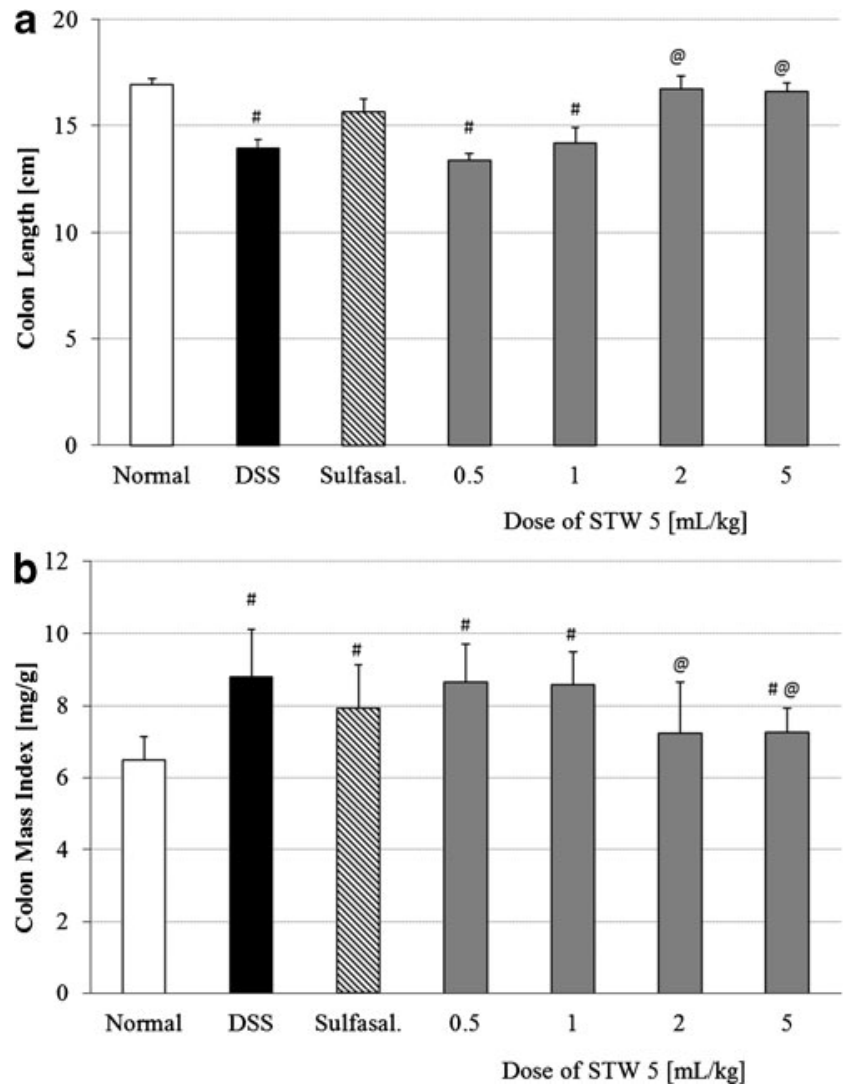

Fig. 2 Effect of STW 5 on colon length (a) and colon mass index (b) in rats with DSS-induced colitis. Data are expressed as means \pm SEM of at least eight animals. ${ }^{\#} P \leq 0.05$ vs. normal control, ${ }^{\circledR} P \leq 0.05$ vs. DSS control 


\section{Results}

Body weight

DSS-induced colitis led to a dramatic inhibition in the rate of weight gain of rats by ca. $70 \%(p<0.001)$. Pretreatment with STW $5(2$ and $5 \mathrm{~mL} / \mathrm{kg})$ tended to restore the normal rate of weight gain $(p=0.09)$ while the lower doses of STW 5 and sulfasalazine $(300 \mathrm{mg} / \mathrm{kg}$ ) had little effect (Fig. 1).
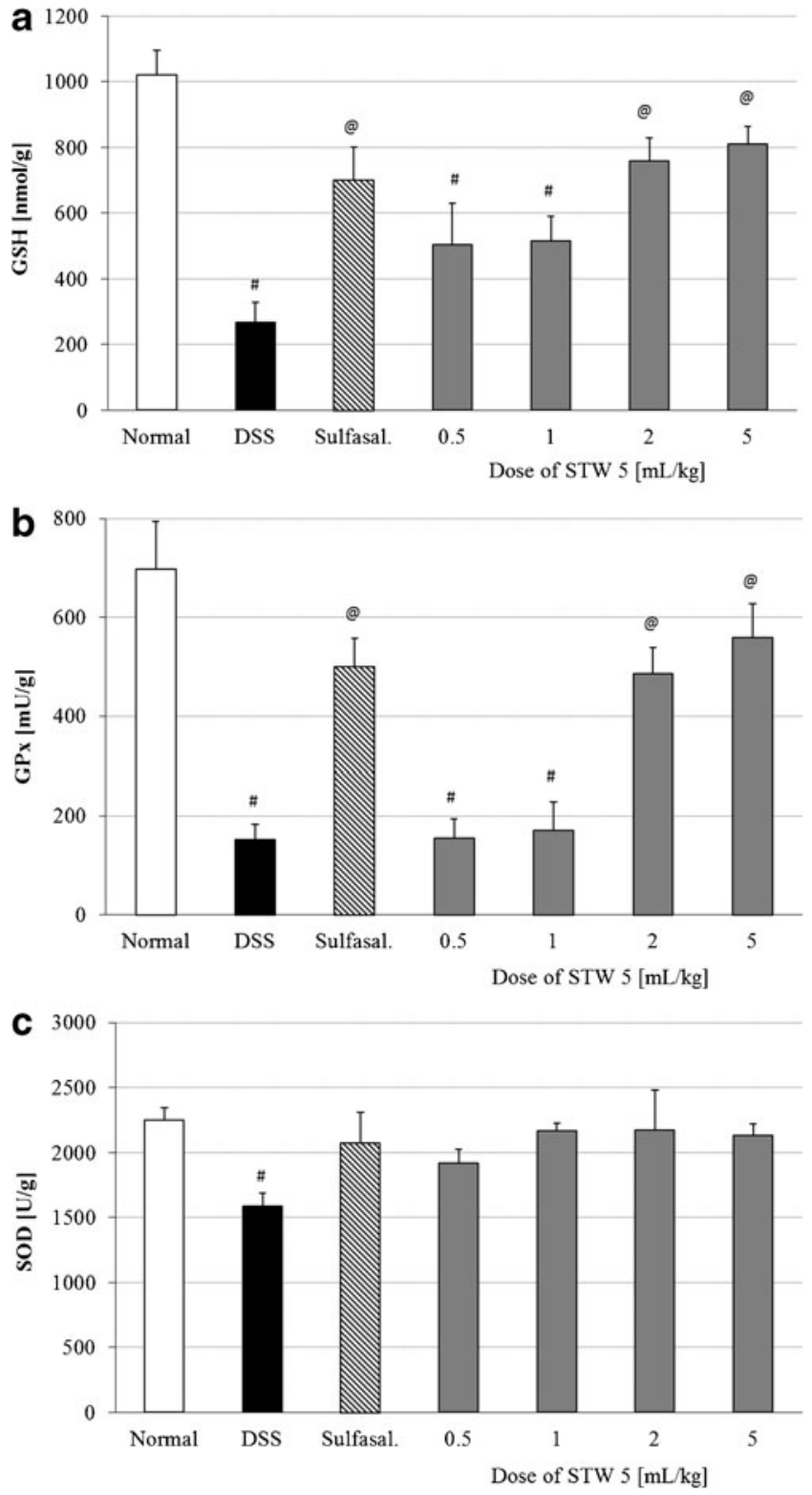

Fig. 3 Effect of STW 5 on reduced glutathione (GSH) levels (a), glutathione peroxidase (GPx) activity (b) and superoxide dismutase (SOD) activity (c) in colonic tissue of rats with DSS-induced colitis. Data are expressed as means \pm SEM of at least eight animals. ${ }^{\#} P \leq 0.05$ vs. normal control, ${ }^{@} P \leq 0.05$ vs. DSS control
Colon length and colon mass index

Induction of colitis led to a reduction in colon length by $17 \%$ as compared to the normal control group (Fig. 2a). This was associated with a $35 \%$ increase in colon mass index (Fig. 2b). Pretreatment with STW 5 tended to prevent these changes in a dose-dependent manner. The effect of the two higher doses of STW 5 was comparable to that of sulfasalazine (Fig. $2 ; p<0.001$ and $p=0.002$ for colon length and colon mass index respectively).
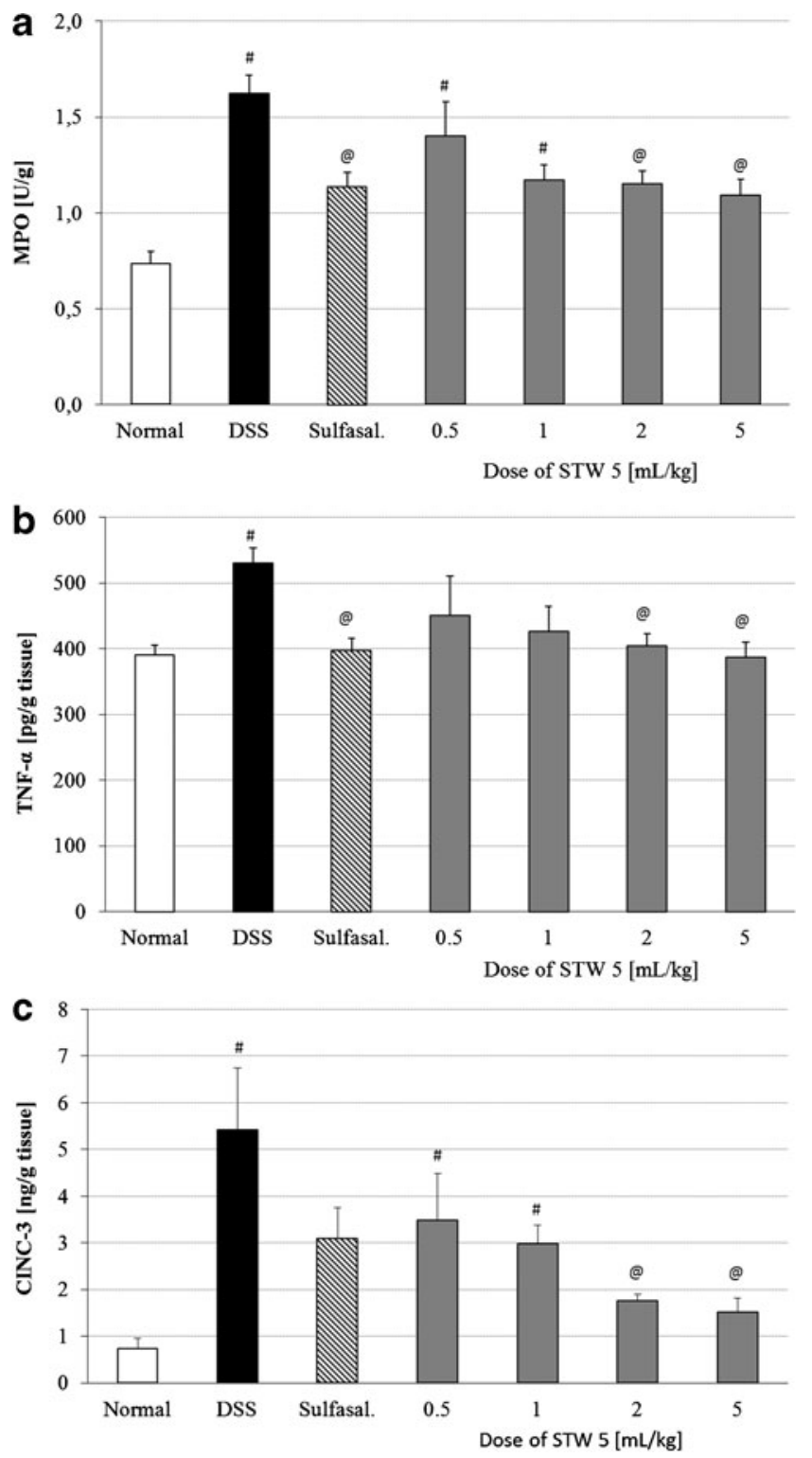

Fig. 4 Effect of STW 5 on inflammatory parameters in colonic tissue of rats with DSS-induced colitis. a Myeloperoxidase $(M P O)$ activity, $\mathbf{b}$ TNF- $\alpha$ levels, $\mathbf{c}$ CINC-3 levels. Data are expressed as means \pm SEM of at least eight animals. ${ }^{\#} P \leq 0.05$ vs. normal control, ${ }^{\circledR} P \leq 0.05$ vs. DSS control 


\section{Biochemical parameters}

The induction of colitis was associated with changes in the various parameters indicative of oxidative stress. This was evidenced by a marked reduction in colonic content of GSH (Fig. 3a) as well as the activities of GPx (Fig. 3b) and SOD (Fig. 3c). Pretreatment with STW 5 dose-dependently reversed the deranged levels of GSH and GPx (Fig. 3a and b, respectively; $p<0.001)$. The reduction in colonic SOD activity induced by DSS was largely prevented by pretreatment with either STW 5 or sulfasalazine (Fig. 3c).

DSS-induced colitis led to a significant increase in MPO activity in the colon, an effect which was largely prevented by pretreatment with either STW 5 or sulfasalazine (Fig. 4a; $p<0.001$ ).

The inflammatory mediators, TNF- $\alpha$ and CINC-3 (Fig. $4 \mathrm{~b}$ and c, respectively), were elevated in colonic tissue of DSS treated rats, but the rise was prevented by pretreatment with either STW 5 or sulfasalazine $(p<0.001)$.

Histopathological examination

The major elements of colonic inflammation include crypt destruction, mucosal ulceration, and infiltration of lymphocytes into the mucosal tissue. Representative histological images of H\&E-stained colon sections from each group are shown in Fig. 5. In contrast to normal control animals (Fig. 5a), DSS-treated animals (Fig. 5b) showed marked necrosis of the epithelium, intraluminal accumulation of mucous exudates, dilatation of the crypts, and submucosal edema. These changes were associated with massive inflammatory cell infiltration in the lamina propria and submucosa. The infiltrated inflammatory cells included polymorphonuclear leucocytes, lymphocytes, and plasma cells.
Fig. 5 Effect of STW 5 on histopathological changes of rat colon in DSS model of colitis. a Normal control rat: normal histological structure of mucosa, b DSS control rat showing necrosis of epithelium, distortion of crypts, inflammatory infiltrate in lamina propria and submucosa as well as submucosal oedema, c sulfasalazine pre-treated rat showing nearly normal structure, d STW $5(2 \mathrm{~mL} / \mathrm{kg})$ pretreated rat showing moderate inflammatory infiltrate in lamina propria and submucosa, e rat pre-treated with STW $5(5 \mathrm{~mL} / \mathrm{kg})$ showing minimal changes. (H\&E staining, $\times 200$ original magnification). $\mathbf{f}$ Total histology score, data are expressed as box plots of the median of at least eight animals. ${ }^{\#} P \leq 0.05$ vs. normal control, ${ }^{\circledR} P \leq 0.05$ vs. DSS control
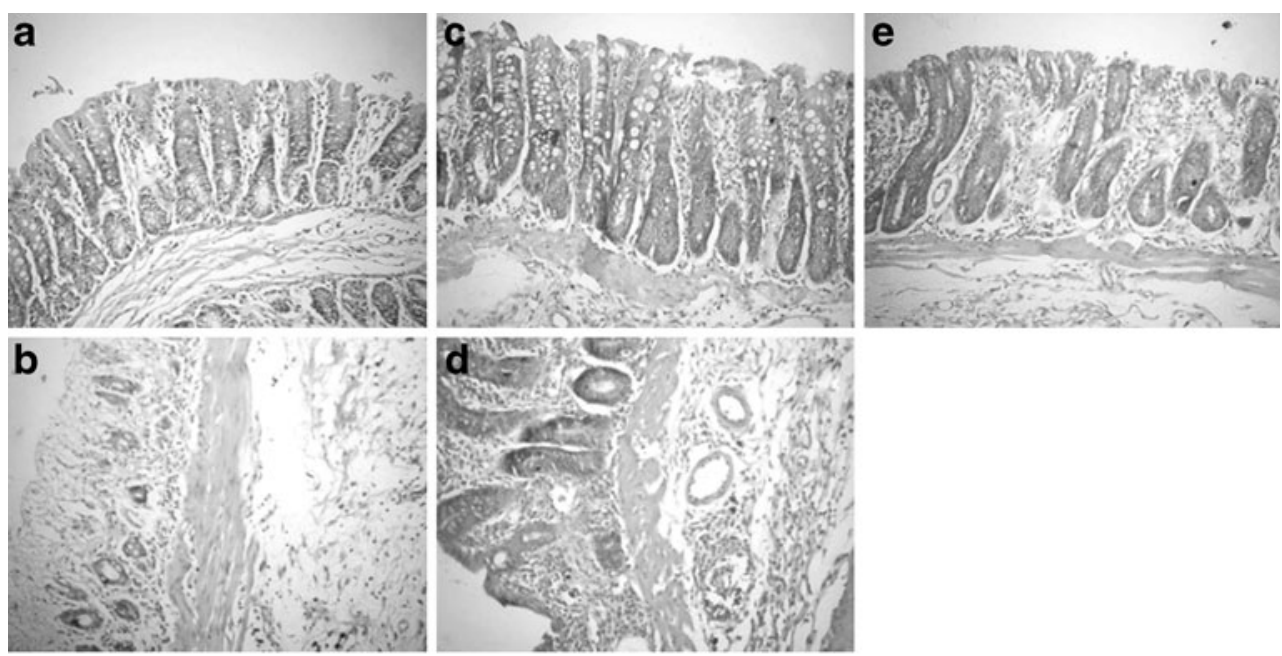

f

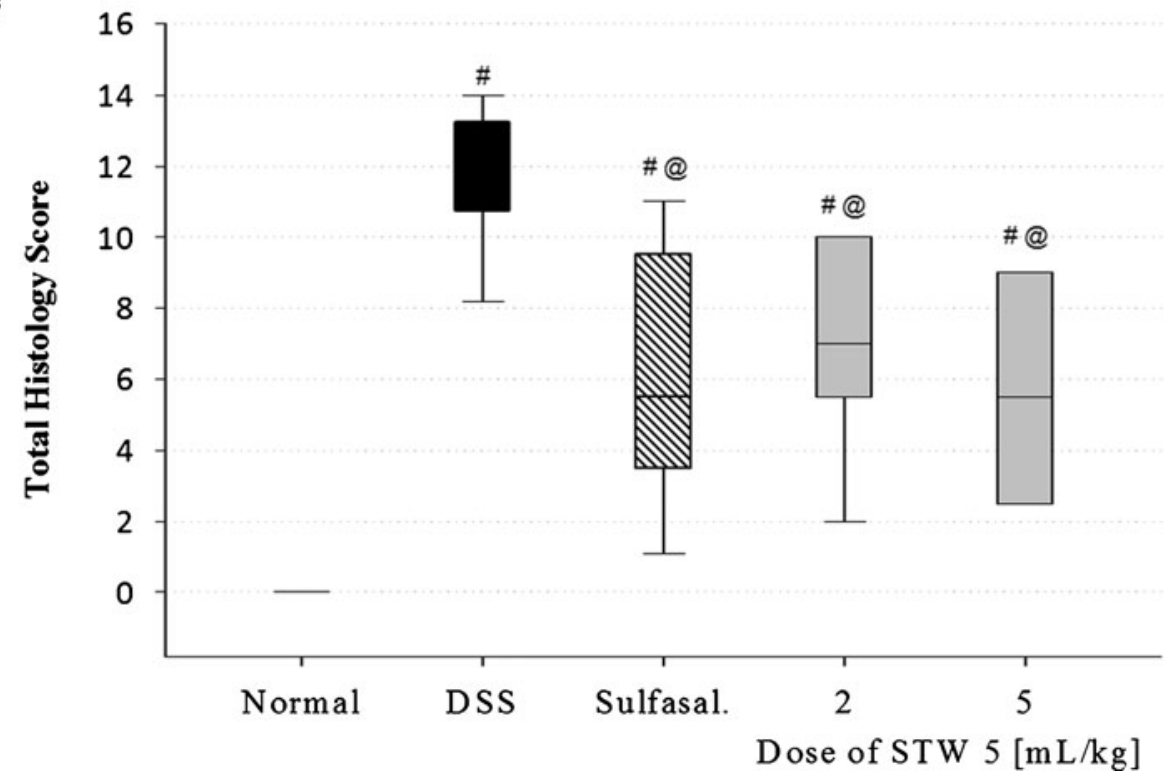


The total histology score was markedly increased in DSS-treated rats (Fig. 5f). Pretreatment with STW 5 largely protected against the histological changes induced by DSS as evidenced by the lesser severity of the above parameters. The inflammatory infiltration in the lamina propria and submucosa was only mild to moderate (Fig. $5 \mathrm{~d}$, e), and the total histology score was markedly decreased (Fig. 5f). The higher the dose of the drug, the greater was its protective effect. The effect of STW 5 was similar to a large extent to the effects of sulfasalazine (Fig. $5 \mathrm{c}, \mathrm{f}$ ).

\section{Colonic motility}

Colonic strips from rats with colitis showed depressed responsiveness to carbachol and $\mathrm{KCl}$, an effect which tended to be prevented by both STW 5 and sulfasalazine (Fig. 6a, b, respectively).

Trypsin $(30 \mu \mathrm{mol} / \mathrm{L})$ was found to cause a $64 \%$ relaxation of the carbachol-induced contraction of the normal rat colon. The extent of relaxation was markedly reduced in colons of rats with DSS-induced colitis, reaching only $24 \%$. Pretreatment with either sulfasalazine or STW 5 tended to restore the relaxing effect of trypsin to reach $58 \%$ and $39 \%$, respectively (Fig. 6c).

\section{Discussion}

The effect of STW 5 on various parameters that have been deranged as a result of inducing colitis through the administration of DSS in rats was studied. Macroscopically, DSS-induced colitis resulted in a marked reduction in the rate of growth of the animals. This feature of DSS colitis resembles the common problem of weight loss observed in IBD patients [12-14]. STW 5 in the higher dose range as well as sulfasalazine tended to restore the rate of weight gain. The effects on weight gain correlated well with the changes in colon length and colon mass index. The DSSinduced increase in colon mass index may be related to the submucosal edema shown histologically while the colon shortening may be associated with its thickening due to edema and infiltration of inflammatory cells into the lamina propria and submucosa $[15,16]$. STW 5 led to marked reduction in the inflammatory infiltrate in both lamina propria and submucosa and dose-dependently protected against changes in colon length and mass index.

Neutrophil infiltration is usually associated with increased MPO activity, a parameter which correlates well with the severity of the lesions in acute DSS-induced colitis [17]. MPO itself possesses cytokine-like properties and can activate neutrophils [18] resulting in the release of a wide range of inflammatory mediators and reactive oxygen species. A decrease in MPO activity after pretreatment with STW 5 is therefore indicative of a reduction in neutrophil
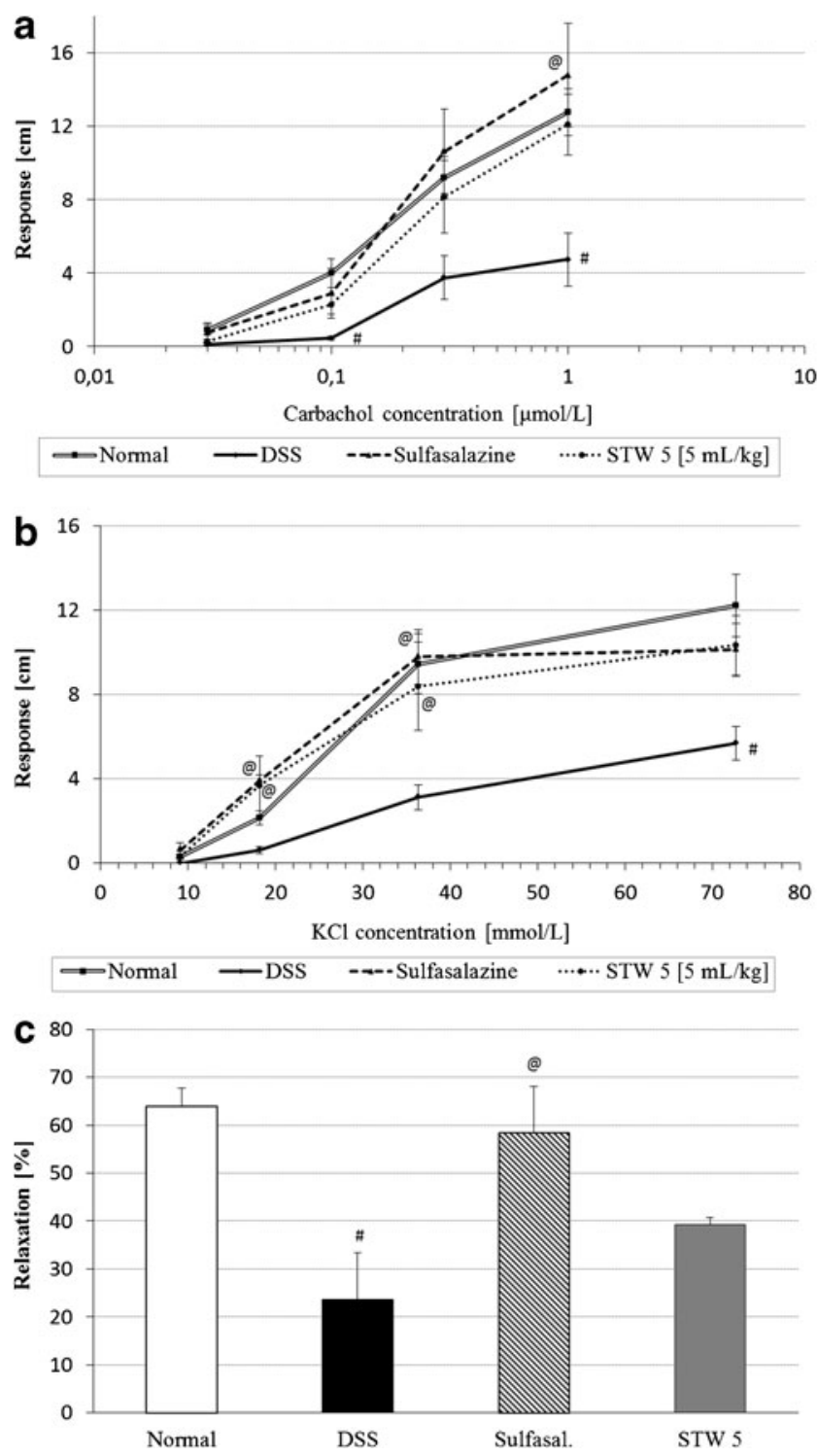

Fig. 6 Effect of STW 5 on the colonic motility changes induced by DSS. a Non-cumulative concentration-response curves to carbachol, $\mathbf{b}$ non-cumulative concentration-response curves to $\mathrm{KCl}$, $\mathbf{c}$ relaxant effect of trypsin after pre-contraction with carbachol [trypsin $(30 \mu \mathrm{mol} / \mathrm{L})$ was applied 5 min after addition of submaximal dose of carbachol]. Data are expressed as means \pm SEM of at least four animals. ${ }^{\#} P \leq 0.05$ vs. normal control, ${ }^{\circledR} P \leq 0.05$ vs. DSS control

infiltration and reduction of the inflammatory process. Similar effects were recently reported in a model of reflux esophagitis [19].

The increased level of oxidative stress in the present study was associated with marked depletion of the cellular antioxidant, GSH as was reported in earlier studies [20,21]. Other related parameters, such as the activities of the two antioxidant enzymes, SOD and GPx were also decreased. Such effects have also been reported in IBD patients [22]. Pretreatment with STW 5 protected dose dependently against the rise in free radical production by DSS, evidenced 
by restoration of colonic GSH, SOD, and GPx levels. Earlier authors reported that STW 5 possesses potent antioxidant activity in several in vitro models by virtue of its radicalscavenging activity and its ability to specifically inhibit metabolic pathways leading to cellular release of free radicals $[23,24]$.

Other factors that may be involved in the inflammatory process in human IBD as well as in DSS colitis include proinflammatory cytokines such as interleukin (IL)- $1 \beta$ and TNF- $\alpha$ [25-27]. Both TNF- $\alpha$ and IL-1 $\beta$ have been implicated in stimulating the production of IL-8 [28], a chemokine implicated in the pathophysiology of IBD [29]. One of the functional analogues of IL-8 in rodents is CINC-3, a potent chemotactic factor for neutrophils. The levels of TNF- $\alpha$ and CINC-3 were significantly raised in the colon following induction of colitis by DSS. Pretreatment with STW 5 dose dependently prevented the rise in both TNF$\alpha$ and CINC-3. The reduction in TNF- $\alpha$ levels by STW 5 , which was also described in several other models of GI inflammation $[8,9,19,30]$, may be explained partly by the previously reported inhibition of the translocation of nuclear factor-kappa B (NF-kB) [31]. NF-kB is believed to play a major role in the regulation of pro-inflammatory gene transcription [32].

Colitis is often associated with bowel motility changes leading to either constipation or diarrhea in patients with IBD $[33,34]$. Colonic contractility has been reported to be deranged also in animal models of colitis [35] and to persist even after resolution of inflammation [36]. Both receptordependent and -independent mechanisms contribute to the altered smooth muscle function during colitis [37]. Colonic tissue from rats with DSS-induced colitis exhibited reduced contractility to both a receptor-dependent muscarinic agonist, carbachol, and a receptor-independent one, $\mathrm{KCl}$, which stimulates smooth muscle directly by depolarizing the intact cell membranes.

The findings are suggestive of a reduced responsiveness of the colon to both parasympathetic stimulation and to direct muscle stimulation by depolarizing agents. Although the myogenic mechanism for decreased motility of inflamed intestinal tissue have not been well examined, some reports suggest that it involves a decreased activity of voltagedependent L-type $\mathrm{Ca}^{2+}$ channels $[38,39]$, an effect that has been shown to be reversed by NF-kB inhibitors [39]. Since STW 5 was shown to inhibit the translocation and hence activation of NF- $\mathrm{KB}$ [31] the observed effects of the drug on restoring normal colonic responsiveness may be due to its anti-inflammatory effect, and hence conservation of L-type $\mathrm{Ca}^{2+}$ channel activity.

On parallel lines, Michael et al. [30] reported that in vitro induction of inflammation in the jejunum and small intestine by intraluminal instillation of TNBS led to a decreased response to acetylcholine, but the response was restored when
STW 5 was instilled concurrently, thereby reducing histopathological inflammatory changes in the mucosa. Earlier studies had shown that STW 5 increased the tone of intestinal or colonic preparations, but had an antispasmodic action when tested against acetylcholine or histamine induced contractions [40], suggesting that STW 5 by virtue of one or more of its components may interact with the muscarinic and/or histaminergic receptors. Studies by Simmen et al. [41] in vitro showed that STW 5 indeed can bind to muscarinic $\mathrm{M}_{3}$ receptors in the intestine as well as to some serotonergic receptors. The modulatory effect of STW 5 may therefore be partially related to up- or downregulation of respective receptors.

As intestinal inflammation is associated with the generation and release of proteases that are potential activators of PAR-2, a receptor known to modulate intestinal motility, identifying the role of PAR-2 in the present model was of interest. Trypsin was found to inhibit carbachol-induced contractions of the normal rat colon. After colonic inflammation induced by DSS, the inhibitory effect of trypsin on carbachol-induced contractions was suppressed. However, pretreatment with STW 5 showed only a tendency to reduce the effect of trypsin.

In conclusion, the present findings show that STW 5 prevents both the colonic inflammatory changes and the motility disturbances in the DSS model of rat colitis. Restoration of normal responsiveness is probably related, at least in part, to the anti-inflammatory properties of the drug. If the present findings can be confirmed in a clinical setting, STW 5 may possibly have some therapeutic usefulness in the management of human IBD. It could be used as an add-on to standard medication to help maintain remission and ameliorate motility disturbances experienced by IBD patients.

Acknowledgements The study was supported by Steigerwald Arzneimittelwerk GmbH, Darmstadt, Germany. The authors wish to acknowledge the help of Dr. Kawkab Abd-El-Aziz Ahmed, Professor of Pathology, Faculty of Veterinary Medicine, Cairo University, for carrying out the histological examinations.

Disclosures O. Kelber and D. Weiser are fully employed by Steigerwald Arzneimittelwerk GmbH, Darmstadt, Germany. H. Abdel-Aziz was not affiliated to that company at the time this study was performed but joined employment as of 9 January 2011. All other authors have no conflict of interest.

Open Access This article is distributed under the terms of the Creative Commons Attribution License which permits any use, distribution, and reproduction in any medium, provided the original author(s) and the source are credited.

\section{References}

1. Collins SM (1996) The immunomodulation of enteric neuromuscular function: implications for motility and inflammatory disorders. Gastroenterol 111:1683-1699 
2. Collins SM, Van Assche G, Hogaboam C (1997) Alterations in enteric nerve and smooth-muscle function in inflammatory bowel diseases. Inflam Bowel Dis 3:38-48

3. Egger B, Bajaj-Elliott M, MacDonald TT, Inglin R, Eysselein VE, Büchler MW (2000) Characterisation of acute murine dextran sodium sulphate colitis: cytokine profile and dose dependency. Digestion 62:240-248

4. Frias R, Ouwehand A, Spillmann T, Vankerckhovend V, HewickerTrautweine M, Salminenc S, Gueimonde M (2009) Effect of clinical and probiotic Lactobacillus rhamnosus strains on intestinal permeability and bacterial translocation in healthy and colitic rats. Food Res Int 42:636-640

5. Rösch W, Liebregts T, Gundermann KJ, Vinson B, Holtmann G (2006) Phytotherapy for functional dyspepsia: a review of the clinical evidence for the herbal preparation STW 5. Phytomedicine 13:114-121

6. Madisch A, Holtmann G, Plein K, Hotz J (2004) Treatment of irritable bowel syndrome with herbal preparations: results of a double-blind, randomized, placebo-controlled, multi-centre trial. Aliment Pharmacol Ther 19:271-279

7. Madisch A, Holtmann G, Mayr G, Vinson B, Hotz J (2004) Treatment of functional dyspepsia with a herbal preparation. A double-blind, randomized, placebo-controlled, multicenter trial. Digestion 69:45-52

8. Khayyal MT, El-Ghazaly MA, Kenawy SA, Seif-el-Nasr M, Mahran LG, Kafafi YA, Okpanyi SN (2001) Antiulcerogenic effect of some gastrointestinally acting plant extracts and their combination. Arzneimittelforschung 51:545-553

9. Khayyal MT, Seif-El-Nasr M, El-Ghazaly MA, Okpanyi SN, Kelber O, Weiser D (2006) Mechanisms involved in the gastroprotective effect of STW 5 (Iberogast) and its components against ulcers and rebound acidity. Phytomedicine 13:56-66

10. Beutler E, Duron O, Kelly BM (1963) Improved method for the determination of blood glutathione. J Lab Clin Med 61:882-888

11. Krawisz JE, Sharon P, Stenson WF (1984) Quantitative assay for acute intestinal inflammation based on myeloperoxidase activity. Assessment of inflammation in rat and hamster models. Gastroenterology 87:1344-1350

12. Shin EK, Kwon H, Kim YH, Shin HK, Kim JK (2009) Chrysin, a natural flavone, improves murine inflammatory bowel diseases. Biochem Biophys Res Commun 381:502-507

13. Klein S, Meyers S, O'Sullivan P, Barton D, Leleiko N, Janowitz HD (1988) The metabolic impact of active ulcerative colitis. Energy expenditure and nitrogen balance. J Clin Gastroenterol 10:34-40

14. Rigaud D, Angel LA, Cerf M, Carduner MJ, Melchior JC, Sautier C, René E, Apfelbaum M, Mignon M (1994) Mechanisms of decreased food intake during weight loss in adult Crohn's disease patients without obvious malabsorption. Am J Clin Nutr 60:775781

15. Geier MS, Butler RN, Giffard PM, Howarth GS (2007) Lactobacillus fermentum BR11, a potential new probiotic, alleviates symptoms of colitis induced by dextran sulfate sodium (DSS) in rats. Int J Food Microbiol 114:267-274

16. Kraft SC, Kirsner JB (1971) Immunological apparatus of the gut and inflammatory bowel disease. Gastroenterol 60:922-951

17. Villegas I, Alarcón de la Lastra C, Orjales A, La Casa C (2003) A new flavonoid derivative, dosmalfate, attenuates the development of dextran sulphate sodium-induced colitis in mice. Int Immunopharmacol 3:1731-1741

18. Lau D, Mollnau H, Eiserich JP, Freeman BA, Daiber A, Gehling UM, Brümmer J, Rudolph V, Münzel T, Heitzer T, Meinertz T, Baldus S (2005) Myeloperoxidase mediates neutrophil activation by association with CD11b/CD18 integrins. Proc Natl Acad Sci U S A 102:431-436
19. Abdel-Aziz H, Zaki HF, Neuhuber W, Kelber O, Weiser D, Khayyal MT (2010) Effect of an herbal preparation, STW 5, in an acute model of reflux oesophagitis in rats. J Pharmacol Sci 113:134-142

20. Arafa HM, Hemeida RA, El-Bahrawy AI, Hamada FM (2009) Prophylactic role of curcumin in dextran sulfate sodium (DSS)induced ulcerative colitis murine model. Food Chem Toxicol 47:1311-1317

21. Camuesco D, Comalada M, Rodríguez-Cabezas ME, Nieto A, Lorente MD, Concha A, Zarzuelo A, Gálvez J (2004) The intestinal anti-inflammatory effect of quercitrin is associated with an inhibition in iNOS expression. Br J Pharmacol 143: 908-918

22. D'Odorico A, Bortolan S, Cardin R, D'Inca' R, Martines D, Ferronato A, Sturniolo GC (2001) Reduced plasma antioxidant concentrations and increased oxidative DNA damage in inflammatory bowel disease. Scand J Gastroenterol 36:1289-1294

23. Germann I, Hagelauer D, Kelber O, Vinson B, Laufer S, Weiser D, Heinle H (2006) Antioxidative properties of the gastrointestinal phytopharmaceutical remedy STW 5 (Iberogast). Phytomedicine 13:45-50

24. Schempp H, Weiser D, Kelber O, Elstner EF (2006) Radical scavenging and anti-inflammatory properties of STW 5 (Iberogast) and its components. Phytomedicine 13:36-44

25. Lee M, Kovacs-Nolan J, Archbold T, Fan MZ, Juneja LR, Okubo T, Mine Y (2009) Therapeutic potential of hen egg white peptides for the treatment of intestinal inflammation. J Funct Foods 1:161-169

26. Triantafillidis JK, Merikas E, Georgopoulos F (2011) Current and emerging drugs for the treatment of inflammatory bowel disease. Drug Des Devel Ther 5:185-210

27. Begue B, Wajant H, Bambou JC, Dubuquoy L, Siegmund D, Beaulieu JF, Canioni D, Berrebi D, Brousse N, Desreumaux P, Schmitz J, Lentze MJ, Goulet O, Cerf-Bensussan N, Ruemmele FM (2006) Implication of TNF-related apoptosis-inducing ligand in inflammatory intestinal epithelial lesions. Gastroenterol 130:1962-1974

28. MacDermott RP (1996) Alterations of the mucosal immune system in inflammatory bowel disease. J Gastroenterol 31:907-916

29. Umehara Y, Kudo M, Nakaoka R, Kawasaki T, Shiomi M (2006) Serum proinflammatory cytokines and adhesion molecules in ulcerative colitis. Hepatogastroenterology 53:879-882

30. Michael S, Kelber O, Hauschildt S, Spanel-Borowski K, Nieber K (2009) Inhibition of inflammation-induced alterations in rat small intestine by the herbal preparations STW 5 and STW 6. Phytomedicine 16:161-171

31. Bonaterra GA, Kelber O, Kinscherf R, Traut U, Seitz S, Weiser D and Metz J (2008) Anti-inflammatory and anti-cancer effects of the phytomedicine STW 5 and its components in vitro. Planta Med 74: PA295

32. Ghosh S, Hayden MS (2008) New regulators of NF-kappaB in inflammation. Nat Rev Immunol 8:837-848

33. Snape WJ Jr, Kao HW (1988) Role of inflammatory mediators in colonic smooth muscle function in ulcerative colitis. Dig Dis Sci 33(Suppl 3):65S-70S

34. Snape WJ Jr, Williams R, Hyman PE (1991) Defect in colonic smooth muscle contraction in patients with ulcerative colitis. Am J Physiol 261:G987-G991

35. Grossi L, McHugh K, Collins SM (1993) On the specificity of altered muscle function in experimental colitis in rats. Gastroenterol 104:1049-1056

36. Wells RW, Blennerhassett MG (2004) Persistent and selective effects of inflammation on smooth muscle cell contractility in rat colitis. Pflugers Arch 448:515-524 
37. Al-Jarallah A, Oriowo MA, Khan I (2007) Mechanism of reduced colonic contractility in experimental colitis: role of sarcoplasmic reticulum pump isoform-2. Mol Cell Biochem 298:169-178

38. Liu X, Rusch NJ, Striessnig J, Sarna SK (2001) Down-regulation of L-type calcium channels in inflamed circular smooth muscle cells of the canine colon. Gastroenterol 120:480-489

39. Kinoshita K, Sato K, Hori M, Ozaki H, Karaki H (2003) Decrease in activity of smooth muscle L-type $\mathrm{Ca} 2+$ channels and its reversal by NF-kappaB inhibitors in Crohn's colitis model. Am J Physiol Gastrointest Liver Physiol 285:G483-G493

40. Ammon HP, Kelber O, Okpanyi SN (2006) Spasmolytic and tonic effect of Iberogast (STW 5) in intestinal smooth muscle. Phytomedicine 13:67-74

41. Simmen U, Kelber O, Okpanyi SN, Jaeggi R, Bueter B, Weiser D (2006) Binding of STW 5 (Iberogast) and its components to intestinal 5-HT, muscarinic M3, and opioid receptors. Phytomedicine $13: 51-55$ 